\title{
On the possibility of low cost, adherent Therapeutic Drug Monitoring in Oncology
}

\author{
Silvia Dalla Marta ${ }^{\mathrm{a}}$, Stefano Fornasaro ${ }^{\mathrm{a}}$, Aleksandra Jaworska ${ }^{\mathrm{a}}$, Giuseppe Toffoli ${ }^{\mathrm{b}}$, \\ Alois Bonifacio $^{\mathrm{a} o}$ and Valter Sergo ${ }^{\mathrm{a} o}$ \\ ${ }^{a}$ Raman Spectroscopy group, Dept. of Architecture and Engineering, University of Trieste, I-34127 \\ Trieste, ITALY; ${ }^{\mathrm{b}}$ Experimental and Clinical Pharmacology Division, Department of Translational \\ Research, IRCCS-National Cancer Institute, Aviano, ITALY; ${ }^{\circ}$ Co-last authors.
}

\begin{abstract}
A frequent quantification of drugs concentrations in plasma of patients subject to chemotherapy is seldom performed, mostly because the standard methods (Gas or Liquid Chromatography coupled with Mass Spectroscopy) are expensive and time consuming.

In this paper we report the approach pursued in one of the research units of the EU project RAMAN4CLINICS to tackle the problem of a low cost, time adherent quantification of drugs used for oncological patients using a Surface Enhanced Raman Scattering (SERS) spectroscopy.

More specifically, the issues concerning the repeatability of the nanostructured substrates will be presented and some promising results to increase the selectivity of the measures toward specific drugs will be discussed, with examples concerning one cytotoxic agent, Irinotecan and one kinase inhibitor, Sunitinib.
\end{abstract}

Keywords: SERS, Therapeutic drug monitoring, paper substrate

\section{INTRODUCTION}

Chemotherapeutic drugs are expensive, have rather heavy side effects on patients, and are usually effective in a very narrow therapeutic window of plasmatic concentrations. All these factors point to the opportunity to optimize their use, adjusting in real time the dosage of the drugs administered to the patients in what is called Therapeutic Drug Monitoring (TDM). Unfortunately, the golden standard for the quantification of drugs in the course of chemotherapeutic treatments is Mass Spectroscopy coupled with High Performance Liquid Chromatography (HPLC-MS), which is both time consuming and expensive. Consequently, TDM is seldom performed on oncological patients and the administration of the drugs is grossly adjusted on the base of the side effects as reported by the patients or as inferred from the routine blood analysis. Simple, rapid and low-cost methods for chemotherapeutic drugs quantification are thus needed to effectively implement TDM. In this contribution we outline an approach that we are pursuing to reach this goal by using a combination of Raman spectroscopy, nanotechnology and chemometrics. Raman spectroscopy in particular has long attracted the interest of the medical community as an analytical tool and it is at the heart of a European initiative called RAMAN4CLINICS, aimed at introducing Raman spectroscopy in clinical practice. One of the activities of RAMAN4CLINICS is specifically devoted to TDM, and the approach used constitutes the backbone of this contribution. When molecules are absorbed on nanostructured metal surfaces, their Raman spectral signature is highly enhanced: this effect is known as Surface Enhanced Raman Scattering (SERS), where the enhancement is up to billions of time (with respect to the spectrum of the same analyte with the same concentration but without adsorption on the nanostructured metal surface). The strong SERS enhancement can be exploited to obtain information from chemotherapeutic drugs in concentration of interest for the oncological patients, typically from nanomolar to micromolar. SERS approaches for drug quantification have been previously tried, also using a separation step different than HPCL (e.g. TLC) before SERS analysis [1]. Once the SERS spectra are obtained, several chemometric techniques can be applied to obtain calibration curves/models capable of establishing appropriate relationships between instrumental responses and the concentration of the drugs of interest in blood/plasma/serum of the patients, which is the ultimate goal.

Biophotonics: Photonic Solutions for Better Health Care V, edited by Jürgen Popp, Valery V. Tuchin,

Dennis L. Matthews, Francesco Saverio Pavone, Proc of SPIE Vol 9887, $98870 \mathrm{G}$

(c) 2016 SPIE $\cdot$ CCC code: 0277-786X/16/\$18 - doi: 10.1117/12.2225232

Proc. of SPIE Vol. 9887 98870G-1 


\section{METHODOLOGY}

A flowchart of the proposed approach in presented in Figure 1. In this section we will present some data for each of the boxes of the flowchart, referring the interested reader to more specific literature for details.

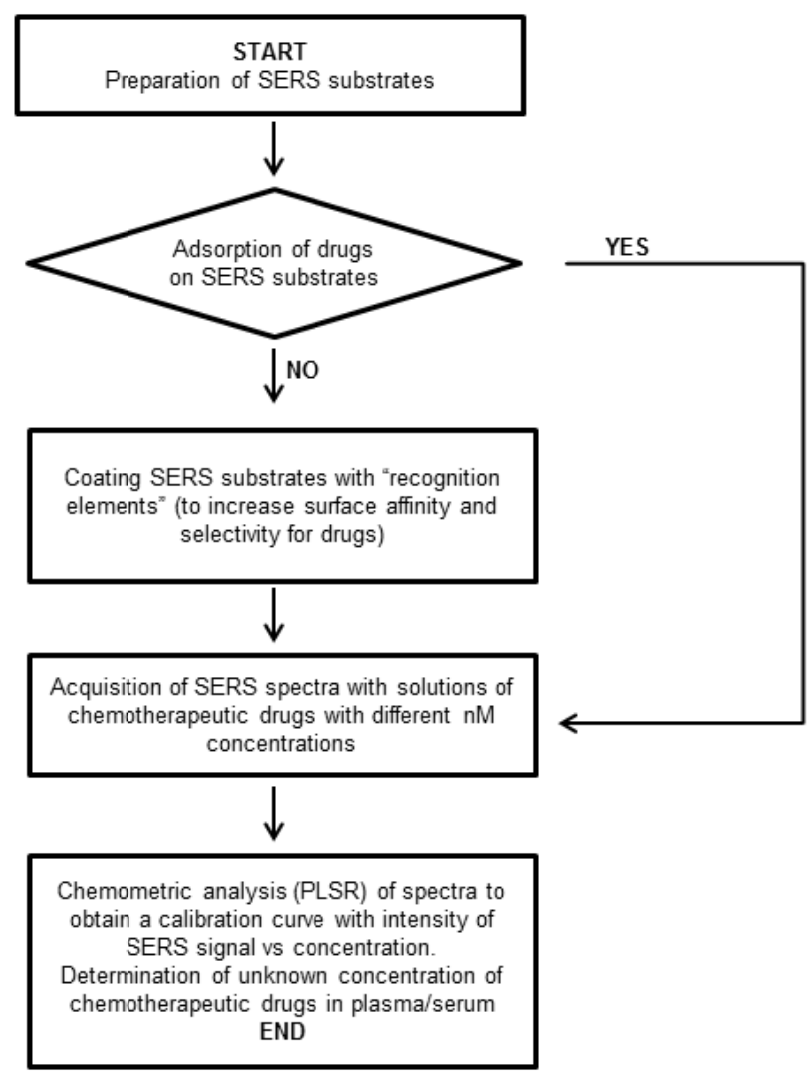

Figure 1 Flowchart of the approach proposed to achieve a TDM of chemotherapeutic drugs through SERS spectroscopy and chemometric analysis.

\subsection{SERS Substrate preparation}

The solid SERS substrates used in this paper were obtained using the dip-coating method [2], loading gold (Au) or silver (Ag) NPs on common filter paper with a $2 \mu \mathrm{m}$ pore size. Au and Ag NPs were synthetized with the Turkevich [3] and Lee Meisel method, respectively [4]. A piece of $1 \mathrm{~cm}^{2}$ was placed on the bottom of a cylindrical glass vial (total capacity of $10 \mathrm{~mL}$ ) containing $3 \mathrm{~mL}$ of $\mathrm{Au}$ or Ag colloidal solution, to which sodium citrate was added up to a final concentration of $20 \mathrm{mM}$. The presence of the citrate leaded to a color change of the colloidal solution, indicating NP aggregation. The vials containing the filter paper were then stocked in the dark at room temperature for one week; after such time, all the NPs were deposited on the vial bottom, covering the paper. The supernatant was extracted with a plastic syringe, paying attention to not touch or move the paper substrate. The substrates were then dried in the vials in air at room temperature and then stocked in Milli-Q water to avoid any loss of NP plasmonic activity. Those substrates feature an intra- and inter-sample repeatability of $15 \%$ for Au NPs and $20 \%$ for Ag NPs. 


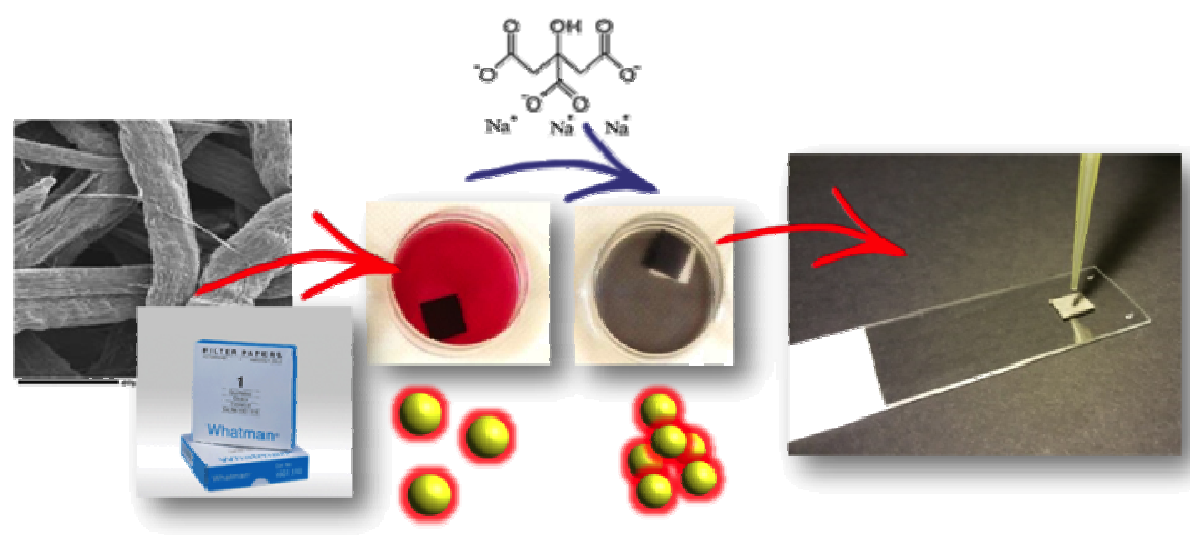

Figure 2 Solid SERS substrate development process.

\subsection{Adsorption of drugs on substrate}

Preliminary SERS measurements of anticancer drugs were performed in Phosphate-Buffered Saline (PBS) solution, a surrogate matrix frequently used to simulate plasma and serum, because its $\mathrm{pH}(7.4)$ and ionic strength $(150 \mathrm{mM})$ are similar to those of the biofluids. The substrates were then incubated with the anticancer drug solution for few minutes; during such time the molecules adsorb on the NPs surface. The substrates were allowed to dry and finally analyzed, acquiring the SERS spectra of the drugs. Figure 3 reports the average SERS spectrum of Irinotecan $270 \mu \mathrm{M}$, plus and minus one intensity standard deviation, acquired on Au solid SERS substrates prepared as described in 2.1. However, the drug concentration detected it is still too high compared with the typical plasmatic values in oncological patient. It is necessary, therefore, to increase the substrates sensitivity especially if the anticancer drug must be detected in complex biofluids such as serum and/or plasma, in which drug molecules must compete with virtually all of the other components of the matrix.

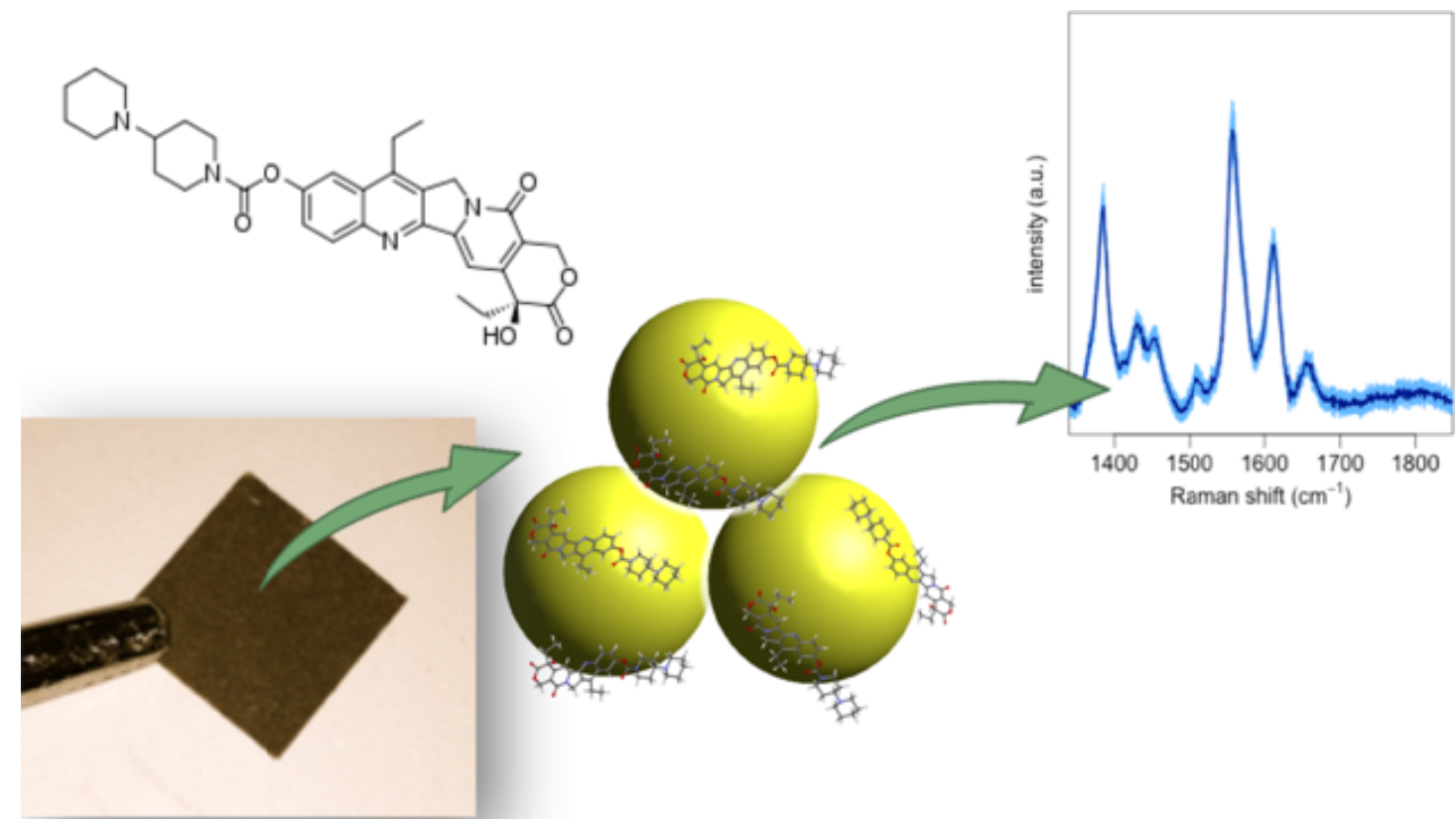

Figure 3 Solid Au SERS substrate image, cartoon of molecular adsorption of NPs surfaces, Irinotecan molecular structure and SERS spectra of Irinotecan in a concentration of $270 \mu \mathrm{M}$, acquired on Au solid SERS substrate. 


\subsection{Coating of SERS substrates with "recognition" elements}

One of the important challenges for application in clinical settings is the improvement of the sensitivity of our SERS substrates to match the therapeutic range of the anticancer drugs (typically down to $0.01 \mathrm{mM}$ ). For this purpose, we functionalized the NPs surfaces, hence the solid SERS substrates, with recognition molecules, exploiting the supramolecular host-guest interactions. Cucurbit[n]urils $(\mathrm{CB}[\mathrm{n}])$, are a family of supramolecular hosts, with a rigid and symmetric macrocyclic. Due to this barrel-like shape, those host structures are able to entrap guest molecules in solution [5]. We exploited these recognition molecules to increase the sensitivity of the substrates for the cytotoxic drug Irinotecan (figure 4). In particular, we report the SERS spectra of the anticancer drug acquired in PBS solutions i) on bare NPs in a concentration of $270 \mu \mathrm{M}$, and ii) the SERS spectra of the same molecule acquired on functionalized NPs with $(\mathrm{CB}[7])$, in a concentration of $2.7 \mu \mathrm{M}$. In the presence of $(\mathrm{CB}[7])$, the anticancer drug can be detected in a concentration of two orders of magnitude lower.

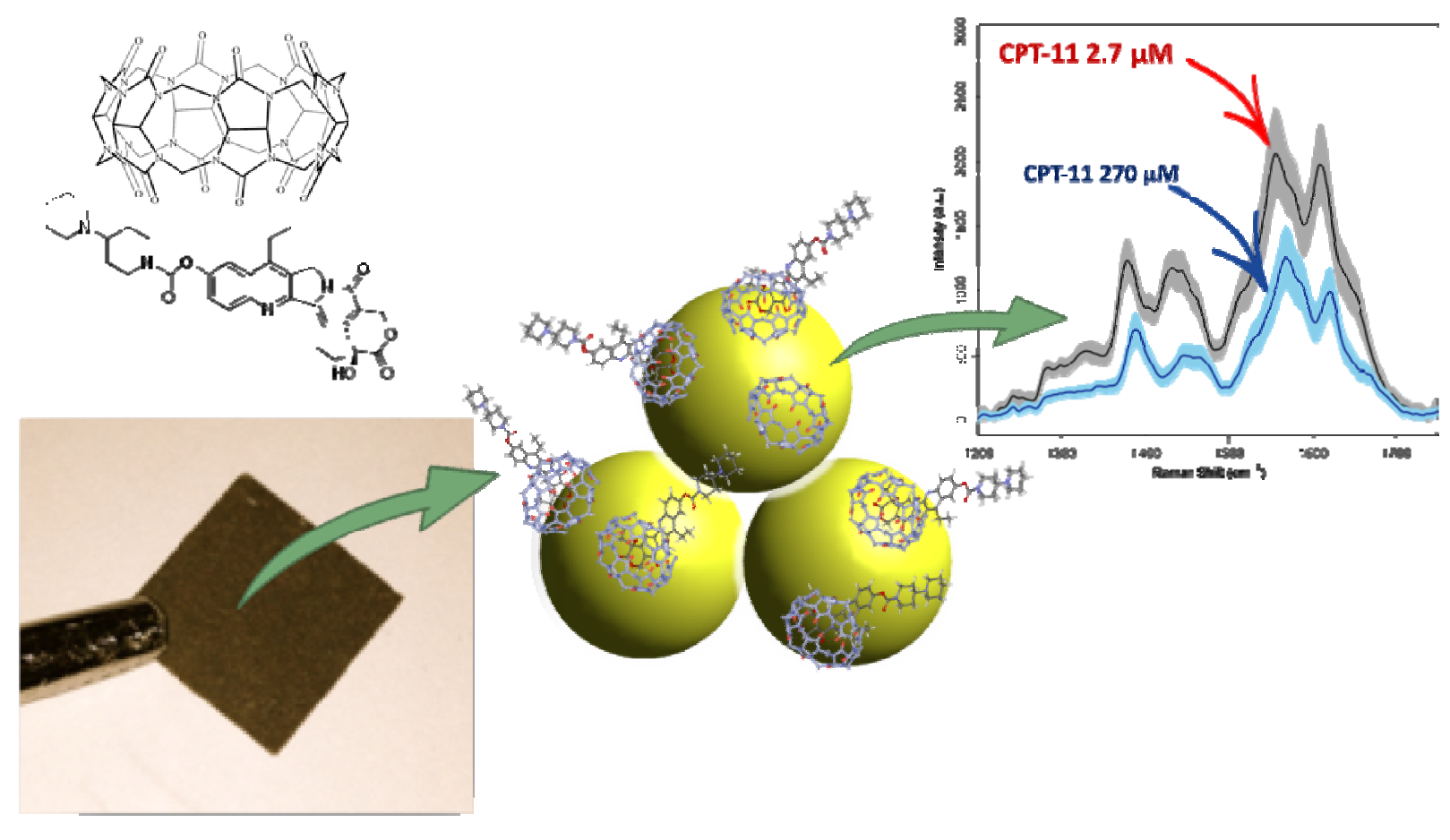

Figure 4 Solid Au SERS substrate image, cartoon of intermolecular interaction of Irinotecan and (CB[7]) and the related adsorption process on NPs surface. Irinotecan and curcubit[7]uril molecular structures. On the left: SERS spectra of Irinotecan in a concentration of $270 \mu \mathrm{M}$, acquired on bare Au solid SERS substrate (in blue) and SERS spectra of Irinotecan in a concentration of $2.7 \mu \mathrm{M}$, acquired on functionalized Au solid SERS substrate (in grey).

\subsection{Chemometric analysis for obtaining calibration models}

To monitor the plasmatic concentration of anticancer drugs in biofluids it is necessary to perform a calibration, through which is possible to extrapolate the information about the concentration of the anticancer drug from SERS spectra. In a calibration, spectra of samples with known concentrations of analytes are measured. In a univariate approach, single intensities (or band areas) are plotted as a function of concentration and a regression is performed relating a measured value to concentration. Although univariate calibration is the most intuitive and simple approach, its application requires the instrumental response to depend only on the concentration of the analyte of interest. Interferences from several 
serum/plasma components may contribute to the measured intensity at a specific Raman shift, making often impossible to differentiate an analyte-specific signal from an interfering one when looking only at one point of a data spectrum. An alternative is a multivariate calibration, using multiple responses simultaneously (e.g., the response at a range of Raman shifts, or over the entire range collected, to calculate concentrations), which may overcome most of the limitations of univariate calibration. In figure 5 we report promising preliminary results of Methotrexate (MTX) quantification in diluted human serum with SERS substrate and chemometric analysis: multivariate calibration models built using partial least-squares regression (PLSR) were compared with univariate calibration. The PLS model outperformed the univariate model.

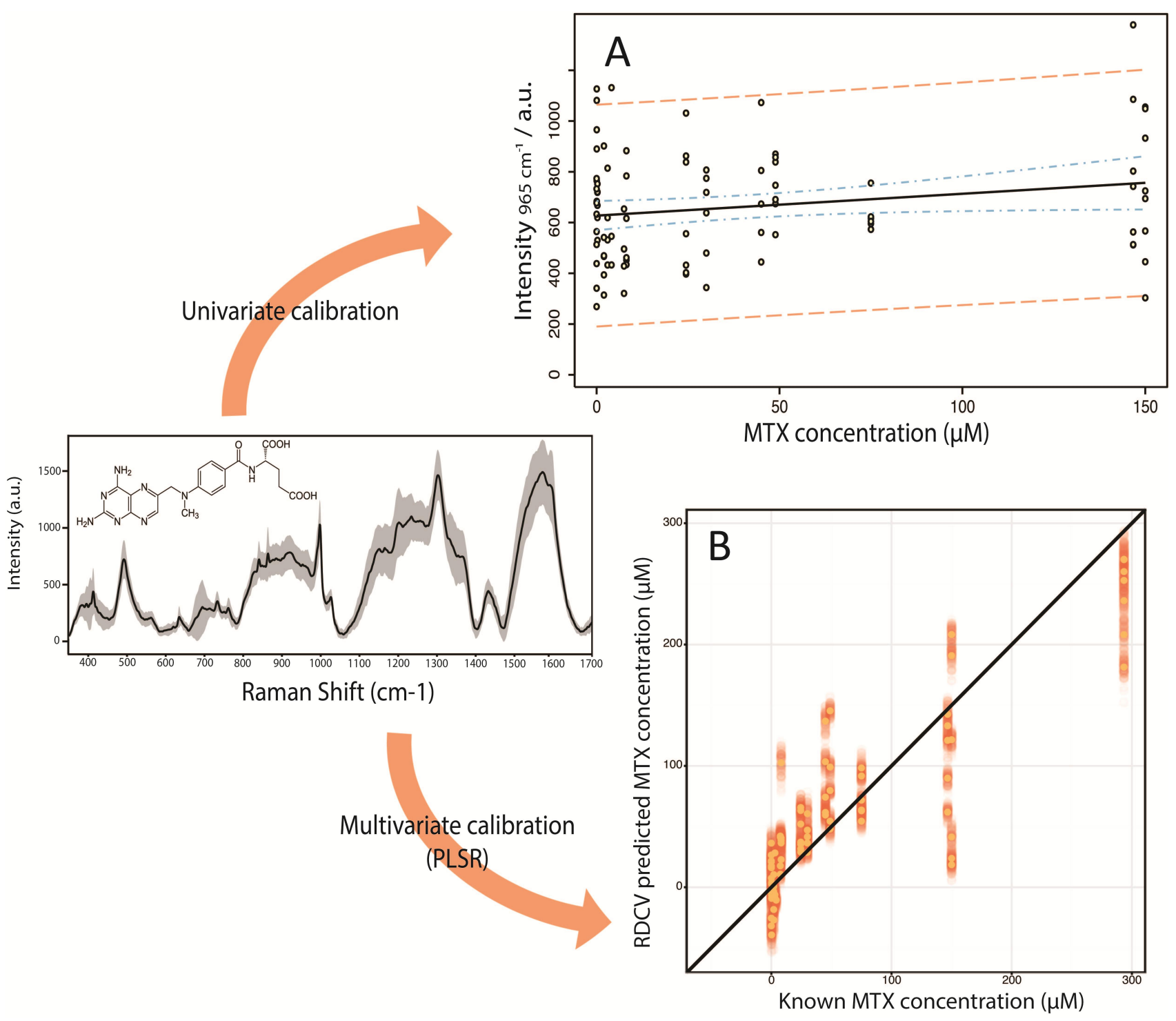

Figure 5 Diagnostic plots from univariate and multivariate calibration for SERS spectra of MTX collected in diluted human serum. A: (o) calibration data for the univariate calibration, confidence bands (broken blue line) and prediction bands (broken red line). B: results from the 100 repetitions in Repeated Double Cross Validation (RDCV) for a Partial Least Square Regression (PLSR) are reported in orange; the means of 91 predictions are reported in yellow. SERS spectrum of MTX $10 \mu \mathrm{M}$ and the chemical structure of MTX are reported for reference. All spectra were collected on functionalized Au solid SERS substrate using an excitation at $785 \mathrm{~nm}$. 


\section{CONCLUSIONS}

In this work, we present solid SERS substrates of Au and Ag NPs, developed with a simple dip-coating method, able to quantify anticancer drugs in a model PBS solution and in a complex matrix, such as diluted human serum, as well if the SERS technique is combined with multivariate calibration. However, the substrates sensitivity its still too low to detect some anticancer agent, such as Irinotecan, in biofluids in therapeutic plasmatic values and moreover, the observed prediction error obtained with multivariate calibration it is still too high if compared with the value provided by established reference methods. Thus, an increase in selectivity and sensitivity of the SERS substrates is necessary. For this reason, we present preliminary results of substrates functionalization using the host-guest supramolecular approach, which increase the substrates sensitivity, at least in a PBS solution, of Irinotecan concentration detection of 2 orders of magnitude. Moreover, we show the advantages of multivariate regression methods with respect to univariate calibration, in the case of MTX.

Overall, this contribution reports the logical procedure used in one of the approaches of the Working Group 1 (Therapeutic monitoring of antitumoral drugs and antibiotic in body fluids) of the EU COST (European Cooperation in Science and Technology) action RAMAN4CLINICS.

\section{AKNOWLEDGMENTS}

SF acknowledges support from IRCCS Burlo Garofolo. AB and, AJ and VS acknowledge support from AIRC (project code 12214), and EU COST action BM1401 (Raman4clinics).

\section{REFERENCES}

[1] A. Vicario, V. Sergo, G. Toffoli et al., "Surface-enhanced Raman spectroscopy of the anti-cancer drug irinotecan in presence of human serum albumin," Colloids and surfaces. B, Biointerfaces, 127C, 41-46 (2015).

[2] L. Polavarapu, and L. M. Liz-Marzan, "Towards low-cost flexible substrates for nanoplasmonic sensing," Phys Chem Chem Phys, 15(15), 5288-300 (2013).

[3] J. Turkevich, P. C. Stevenson, and J. Hillier, "A study of the nucleation and growth processes in the synthesis of colloidal gold,” Discuss. Faraday Soc., 11(0), 55-75 (1951).

[4] P. C. Lee, and D. Meisel, "Adsorption and surface-enhanced Raman of dyes on silver and gold sols," The Journal of Physical Chemistry, 86(17), 3391-3395 (1982).

[5] S. Mahajan, T. C. Lee, F. Biedermann et al., "Raman and SERS spectroscopy of cucurbit[n]urils," Phys Chem Chem Phys, 12(35), 10429-33 (2010). 